\title{
Aimé Césaire : La Quête du « Nègre fondamental »
}

\section{Albert James Arnold}

URL : http://journals.openedition.org/coma/635

DOI : $10.4000 /$ coma.635

ISSN : 2275-1742

\section{Éditeur}

Institut des textes \& manuscrits modernes (ITEM)

\section{Référence électronique}

Albert James Arnold, « Aimé Césaire : La Quête du « Nègre fondamental » », Continents manuscrits [En ligne], 6 | 2016, mis en ligne le 31 octobre 2016, consulté le 01 mai 2019. URL : http:// journals.openedition.org/coma/635; DOI : 10.4000/coma.635

Ce document a été généré automatiquement le 1 mai 2019.

\section{(c) (i) (9)}

Continents manuscrits - Génétique des textes littéraires - Afrique, Caraîbe, dispora est mis à disposition selon les termes de la licence Creative Commons Attribution - Pas d'Utilisation Commerciale - Pas de Modification 4.0 International. 


\title{
Aimé Césaire : La Quête du « Nègre fondamental »
}

\author{
Albert James Arnold
}

\section{NOTE DE L'ÉDITEUR}

Cet article est une reprise, avec l'aimable autorisation de l'auteur et de l'éditeur, du texte paru dans la revue en ligne Pluton Magazine, le 5 février 2016.

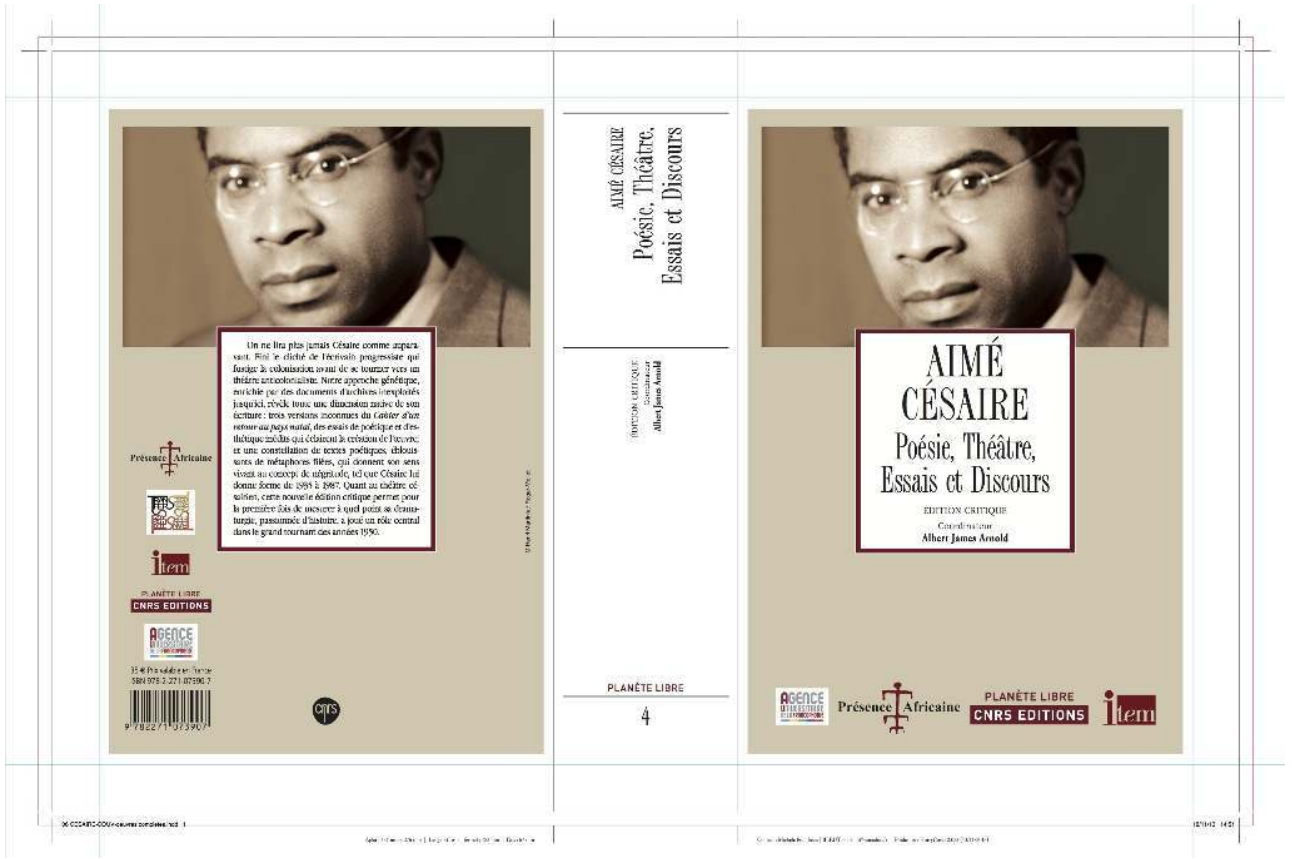

Première de couverture

Crédits : CNRS Editions 
« En profondeur, je me considère comme un poète et que la politique, c'est accidentel $»^{1}$

Doit-on sortir Césaire de la "réserve » des études «francophones » qui le cantonnent dans la région idéologiquement circonscrite $\mathrm{du}$ « Sud»? Les mots entre guillemets sont loin d'être innocents. Depuis plus d'un demi-siècle, les pontes de l'université - en Europe, en Afrique et en Amérique du Nord - interrogent l'œuvre d'Aimé Césaire autour de l'axe unique de la décolonisation et ses séquelles. Au moment de son décès en 2008, les journalistes ont déploré la perte du " chantre de la négritude " sans se demander à quoi cette phrase creuse pouvait se référer. Le lecteur non averti pouvait croire qu'Aimé Césaire était un poète de la Françafrique et que son œuvre appartenait à une ère révolue. Sa panthéonisation par le président Sarkozy - que Césaire avait refusé de recevoir quand Sarko était ministre de l'Intérieur - a achevé ce long processus d'instrumentalisation. Les lecteurs attentifs savent pourtant que le verbe de Césaire est universalisant, quoique centré sur «cette unique race " sortie de la traite et du circuit triangulaire. L'édition à vocation génétique de l'œuvre littéraire de Césaire que j'ai eu l'honneur de coordonner pour la collection Planète Libre, parue à l'occasion du centenaire du poète, mettait déjà les pendules à l'heure ${ }^{2}$. Mon équipe pouvait espérer renverser la vapeur par une contextualisation plus large de son œuvre.

2 Est-ce qu'on réduit la poésie de Victor Hugo à ses engagements politiques? Est-ce qu'on lit celle de Rimbaud à la lumière de la traite des armes entre Aden et le Harar? Commençons à rendre le même honneur et le même respect à la poésie de Césaire !

3 Au lieu de souscrire à la fiction que l'édition du Cahier publiée par Présence Africaine en 1956 fût "définitive », mon équipe a démontré qu'elle représentait une déviation tactique - dans l'intérêt des indépendances africaines - d'une poétique que Césaire élaborait tout au long des années 1940.

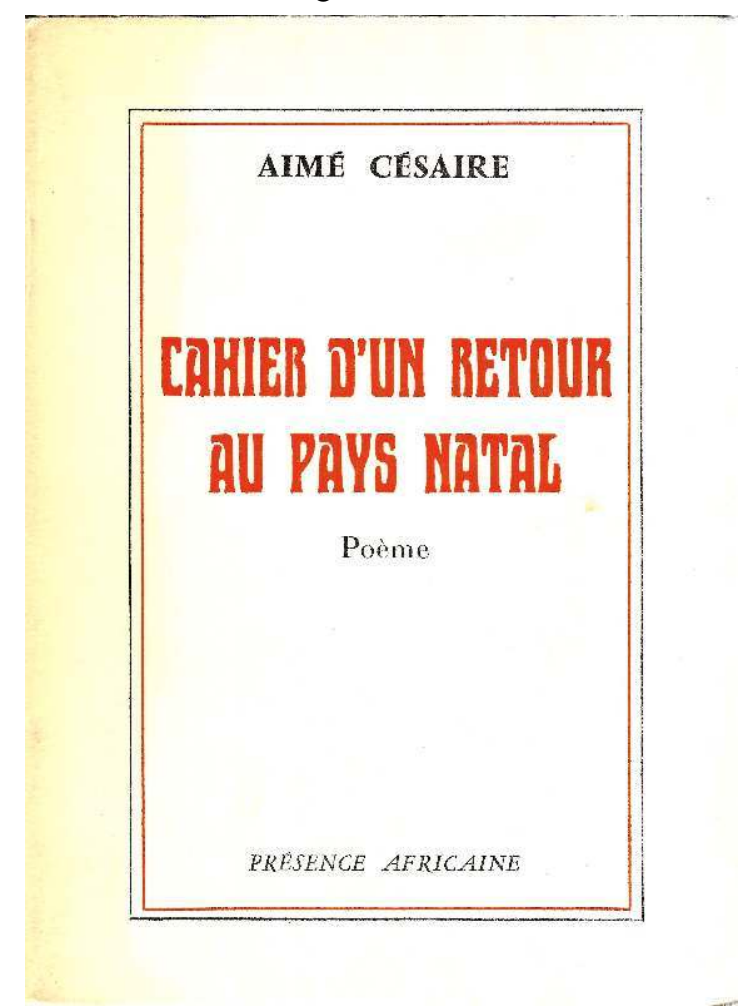

Première de couverture 
La quatrième de couverture de l'édition Présence Africaine a été la première à orienter le lecteur vers une africanisation du Cahier :

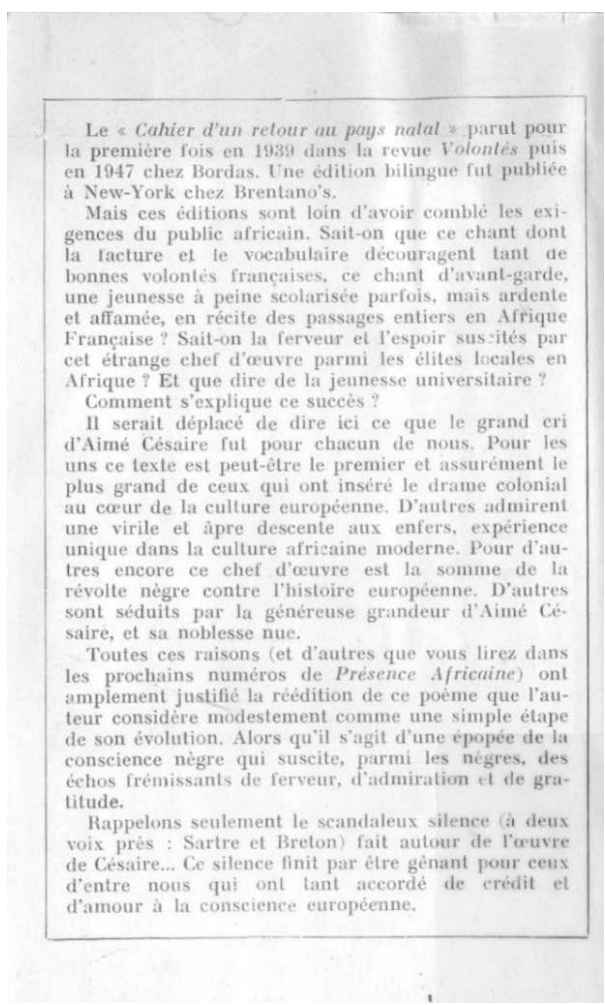

Quatrième de couverture

$5 \quad$ Notre édition présente la première version publiée de chaque texte ou recueil de poésie. Ainsi, au lieu de prêter au poète de 1939 des intentions qui n'appartiennent qu'au tribun de 1955-1956 - soucieux surtout de sensibiliser le lectorat de la nécessité de décoloniser le continent africain - nous interrogeons le texte du Cahier élaboré de 1935 à 1938 dans une longue période d'angoisse existentielle. Dépouillé de tous les ajouts de 1956, le texte de la revue Volontés, édité à la veille de la seconde guerre mondiale, révèle la guérison spirituelle d'une maladie culturelle (la «pseudomorphose» du texte) qui résulte de l'ingurgitation d'une vision du monde impérialiste. Cette version primitive du texte présente une charpente quadripartite qui envisage la mort spirituelle du "bon nègre » fidèle à son «bon maître » et l'émergence d'un être transformé, le " mauvais nègre » qui se promet d'être la bouche de ceux qui n'ont pas de bouche. Au final, ce poète-prophète imagine son peuple, tel Jésus descendu de sa croix et déposé, rompu, entre ses bras. Les références majeures de cette première période sont Péguy et Claudel (pour le verset), Frobenius et Spengler (pour l'ethnographie et l'historiographie) et surtout les prophètes de l'Ancien Testament ${ }^{3}$. Combien de lecteurs du Cahier savent qu'en 1939 Césaire pouvait écrire, au vers qui suit «car pour me cantonner dans cette unique race » "vous savez pourtant mon amour catholique $»^{4}$. 


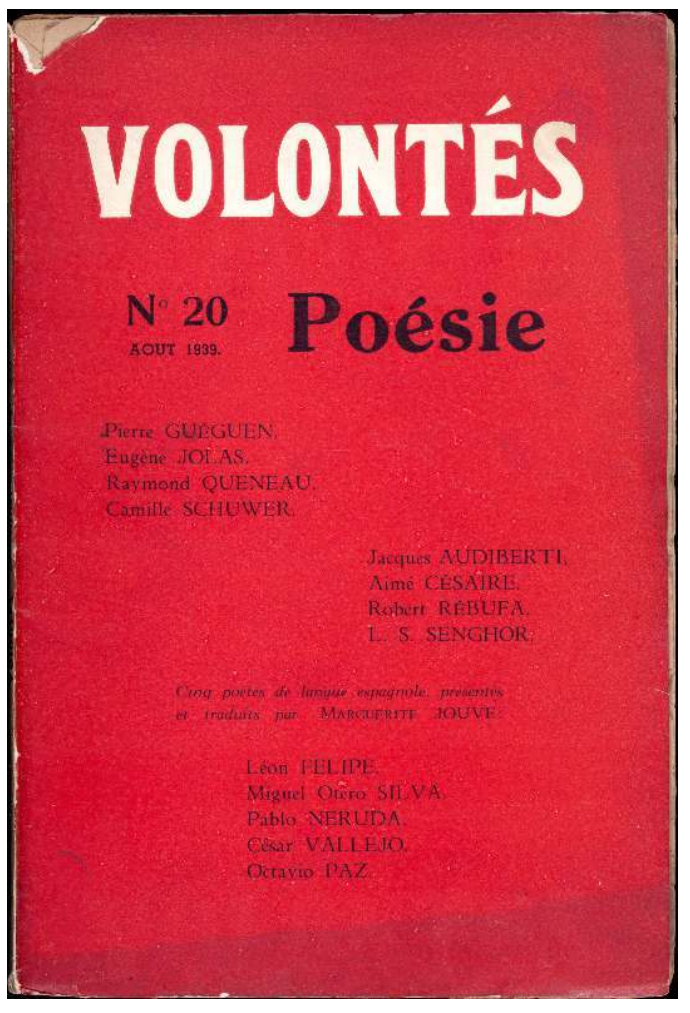

Publication du Cahier en revue, août 1939

6 Ce premier "Cahier » a paru à côté du poème de Senghor "Aux tirailleurs sénégalais morts pour la France » dans la guerre de 14-18, ce qui ne surprendra personne. Vue sous l'angle de l'expérimentation poétique de Césaire entre 1941 et 1945, la présence dans ce même numéro de poèmes de Pablo Neruda, César Vallejo et Octavio Paz est plus intéressante. Comme l'on sait, l'occupation du nord de la France par l'Allemagne nazie a fait replier dans la zone dite libre des centaines d'artistes, d'écrivains et d'intellectuels qui ne plaisaient pas plus à Vichy qu'à l'occupant. L'humanitaire américain Varian Fry a réussi à faire s'évader légalement - au moyen de visas délivrés par des consulats plus ou moins complaisants - beaucoup d'entre eux, y compris les surréalistes de la Villa Air-Bel. Le bateau Capitaine-Paul-Lemerle devait transborder, dans des conditions d'inconfort considérable, Claude Lévi-Strauss, Victor Serge et Anna Seghers, ainsi que les surréalistes André Breton et sa compagne Jacqueline Lamba, Wifredo Lam et sa future épouse Hélène Holzer. Le peintre André Masson, parti de Marseille une semaine plus tard, allait les rejoindre en Martinique. En avril 1941, la rencontre de Césaire avec André Breton a eu sur le jeune poète martiniquais un effet déterminant. La revue Tropiques, et notamment les contributions de Suzanne Césaire et René Ménil, mettent en relief la nouvelle expérimentation du rédacteur en chef. Loin d'être une simple question d'« écriture automatique » comme le prétendent les défenseurs de la lecture politique de Césaire, le surréalisme lui offrait une nouvelle liberté de plume et l'espoir d'un accès à l'inconscient collectif des nègres de la diaspora. Cette thèse s'articule clairement en 1944, dans « Poésie et Connaissance ", texte publié d'une intervention au congrès d'épistémologie tenu à Port-au-Prince en Haïti. Cependant entre 1941 et 1944 nous voyons les nouvelles expérimentations poétiques de Césaire publiées dans les revues éditées par les surréalistes éparpillés par la guerre aux quatre coins de l'hémisphère occidental. Ce sont les premières versions de la majorité des poèmes publiés dans Les armes miraculeuses en 1946. À interroger les revues surréalistes ou surréalisantes de New York, Mexico, 
Santiago du Chili ou Buenos Aires, on découvre une première carrière américaine de Césaire, enfouie et donc oubliée aujourd'hui, qui précède de peu sa carrière française. Rimbaud est certainement le point de départ, comme l'on sait, mais il est tôt relayé par Lautréamont. Pour l'accès à l'inconscient par l'écriture, Césaire se tourne vers Freud, bien sûr, mais aussi vers les archétypes de Jung qu'il pouvait connaître par Pierre Mabille et le Bachelard de la Psychanalyse du feu.

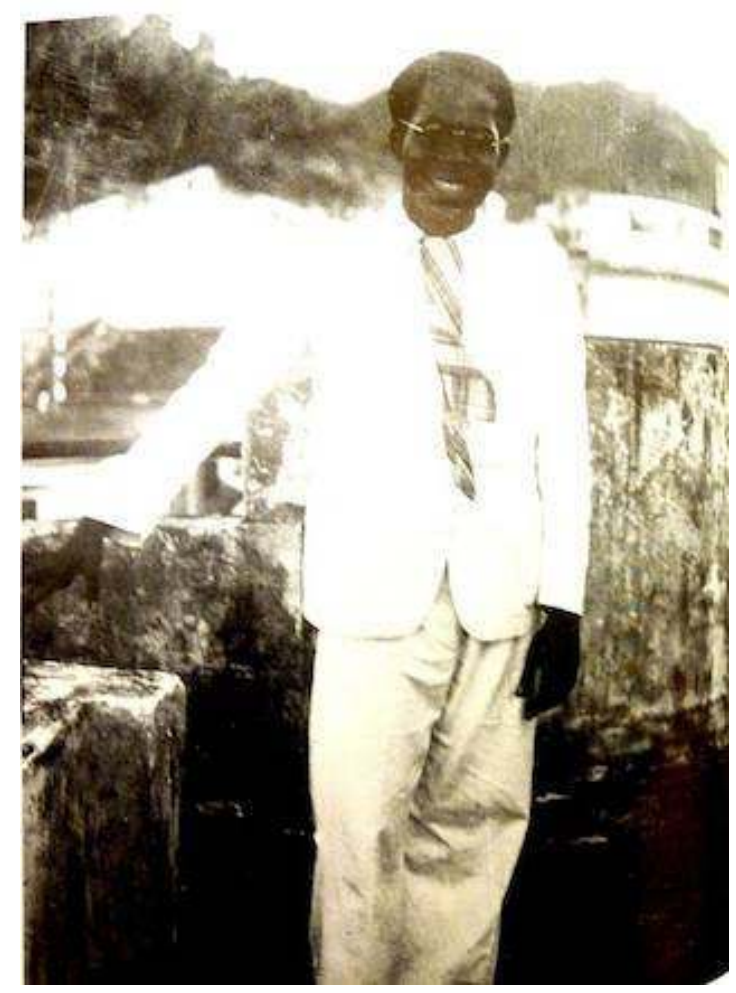

Photo d'Aimé Césaire, février 1944, publiée dans VVV

7 Cette nouvelle phase d'expérimentation poétique comportait un autre élément de taille : Césaire troquait le système référentiel du Cahier de 1939 contre un vaste répertoire de mythes qu'il concevait, à l'instar de l'auteur du Rameau d'or, comme un réservoir de métaphores de mort spirituelle et de renaissance héroïque. Nous en trouvons les traces parmi les poèmes des Armes mais articulées plus solidement dans le mythe tragique qui sous-tend le texte de «Et les chiens se taisaient ». Formant la seconde moitié des Armes de 1946 à 1970, cet oratorio lyrique, désigné « tragédie » par son auteur, jette une lumière insoupçonnée sur l'intention formelle des poèmes qui le précèdent. En 1981 Césaire réaffirmait son accord avec la théorie de Nietzsche selon laquelle le héros tragique est un « être souffrant, et agissant, ou agissant et souffrant, à l'image de Dionysos lui-même ». $\mathrm{Et}$, sur le rôle esthétique et social de la tragédie il rejoint Nietzsche en affirmant que "l'idée de la tragédie, contrairement aux apparences, n'est jamais pessimiste, parce que, par-delà les échecs, par-delà la mort, c'est quand même l'adhésion à la vie » ${ }^{5}$. L'éditeur de ce premier recueil doit fouiller dans les boyaux du texte pour reconnaitre les métaphores qui s'y réfèrent. Il va sans dire que les lectures politiques des Armes sont passées à côté d'allusions qui, dans leur perspective, ne faisaient pas sens. Ce que nous avons trouvé dans «Et les chiens se taisaient ", ce sont de multiples images se rapportant aux dieux de la végétation - Osiris, Attis, Adonis - qui doivent mourir pour que la communauté renaisse. Cette renaissance sociale ne passe pas par une révolution politique mais par une 
secousse - ce que Frobenius appelait un «saisissement»- collective. Les Armes miraculeuses, amputées de leur ancrage dans "Et les chiens se taisaient" à partir de l'édition Désormeaux de 1976, ne sont plus lisibles dans ce même sens. Depuis peu des spécialistes de la poésie moderne ont insisté sur le rôle fondamental que «Et les chiens se taisaient » jouait dans la composition du premier recueil de Césaire ${ }^{6}$. Il est désormais possible d'y reconnaître une intention formelle qui place le poète martiniquais à côté de poètes majeurs du mouvement moderne - Yeats, Pound, Williams entre autres - qui ont cherché à renouveler l'écriture poétique en s'appuyant sur la relecture des mythes de l'antiquité.

8 Une fois avertis des options déterminantes de sa première poétique, nous sommes à même d'aborder l'édition originale (1948) de Soleil cou coupé, que le poète a lui-même éviscérée afin de produire une version politiquement correcte en 1961. Nous venons de nommer le recueil Cadastre, qui s'est aligné sur le Discours sur le colonialisme dans le but de libérer le «continent noir ». La réécriture du poème "À l'Afrique » entre 1948 et 1961 éclaire merveilleusement l'originalité du verbe césairien dans Soleil cou coupé et son édulcoration regrettable dans Cadastre.

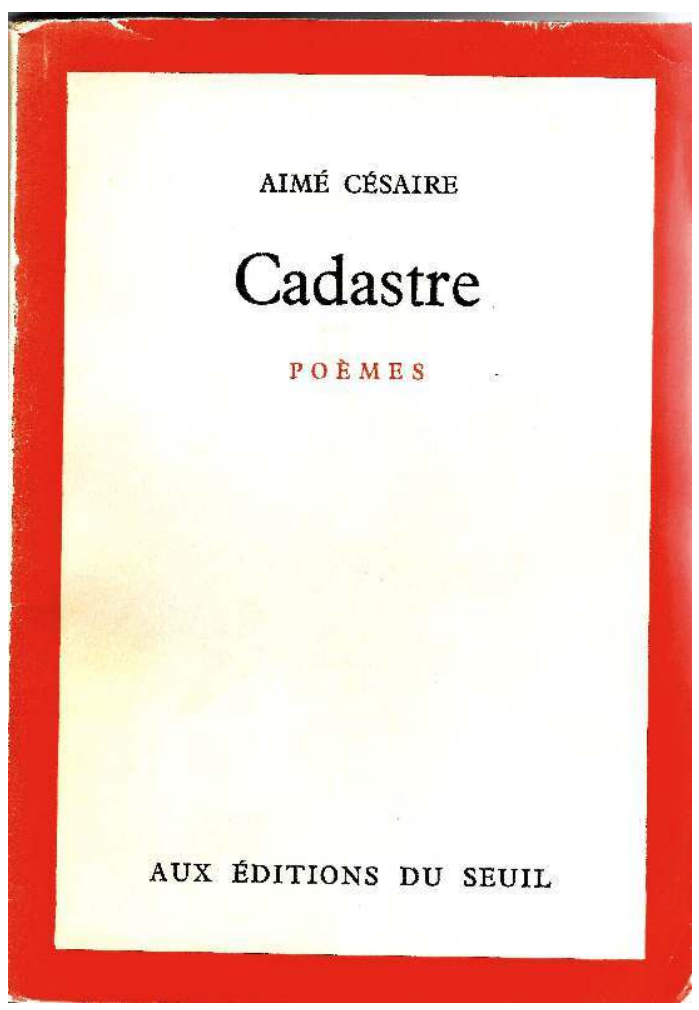

Cadastre, 1961, Seuil éditeur

Depuis 1961 on lit le début de « À l'Afrique » ainsi :

Paysan frappe le sol de ta taba

dans le sol il y a une hâte que la syllabe de l'événement ne dénoue pas

je me souviens de la fameuse peste

il n'y avait pas eu d'étoile annoncière

mais seulement la terre en un flot sans galet pétrissant d'espace

un pain d'herbe et de réclusion

frappe paysan frappe

le premier jour les oiseaux moururent

le second jour les poissons échouèrent 
le troisième jour les animaux sortirent des bois

et faisaient aux villes une grande ceinture chaude très forte

Dans cette version le temps historique domine, marqué par le passé simple des verbes. Le paysan est un travailleur colonial vu sous un angle tiers-mondiste. La «hâte » du deuxième vers doit forcément appeler l'indépendance imminente de l'Afrique.

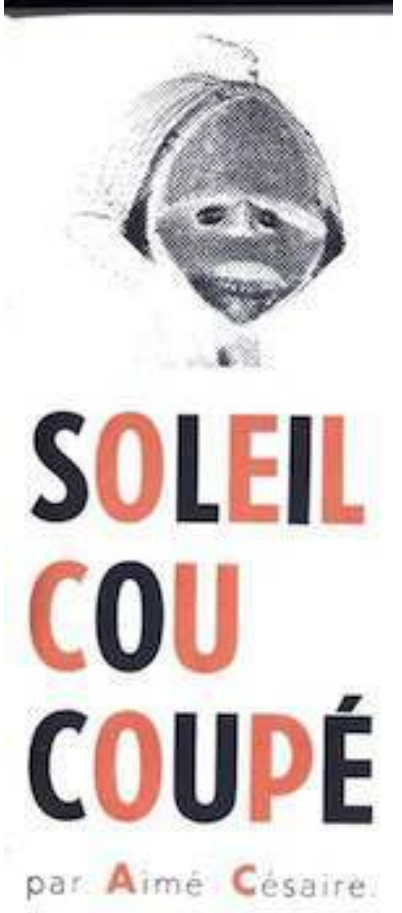

Première de couverture, Soleil cou coupé, 1948

Or, la version de 1948 était tout autre et relevait d'une vision du monde non politique mais spirituelle au sens large :

Paysan frappe le sol de ta daba

dans le sol il y a une hâte que la syllabe de l'événement de dénoue pas

je me souviens de la fameuse peste qui aura lieu en l'an 3000

il n'y avait pas eu d'étoile annoncière

mais seulement la terre en un flot sans galet pétrissant d'espace un pain

d'herbe et de réclusion

frappe paysan frappe

le premier jour les oiseaux mourront

le second jour les poissons échouèrent

le troisième jour les animaux sortirent des bois

et faisaient aux villes une grande ceinture chaude très forte

12 L'interpénétration des temps du verbe (futur, présent, passé) situe l'action du paysan dans une intemporalité propre au mythe, non à l'histoire. L'absence d'étoile annoncière suggère une culture religieuse, non une réalité socio-économique. Le lien implicite entre l'action du paysan et des événements tels que la peste biblique place la scène dans le merveilleux, non dans le réel. Un long passage mène au refus de la religion de l'Occident et à une épiphanie. L'Afrique que le poème évoque se situe de l'autre côté d'une apocalypse à venir ${ }^{7}$ :

Paysan le vent où glissent des carènes arrête autour de mon visage la main lointaine d'un songe

ton champ dans son saccage éclate debout de monstres marins 
que je n'ai garde d'écarter

et mon geste est pur autant qu'un front d'oubli

Cette Afrique n'est nullement le continent colonisé tel qu'il existait en 1946 et le paysan que Césaire y place n'est pas un simple laboureur. Au contraire, pourrait-on dire, cette Afrique est l'image de l'espérance et la promesse d'une transformation spirituelle. En cela elle ressemble à l'idéal qui traverse la poésie de Césaire de 1939 à 1950 environ. Elle baigne dans un climat d'épiphanies liminales ponctuées par la dénonciation des méfaits $\mathrm{du}$ colonialisme et cela dans un langage qui rappelle le plus souvent les récriminations des prophètes de l'Ancien Testament. Tout cela change entre 1950 et 1956, date de la réédition du Cahier d'un retour au pays natal qui vide le poème de toutes ses attaches spirituelles et de sa facture surréaliste, introduite d'ailleurs à l'époque où Césaire composa « À l'Afrique ».

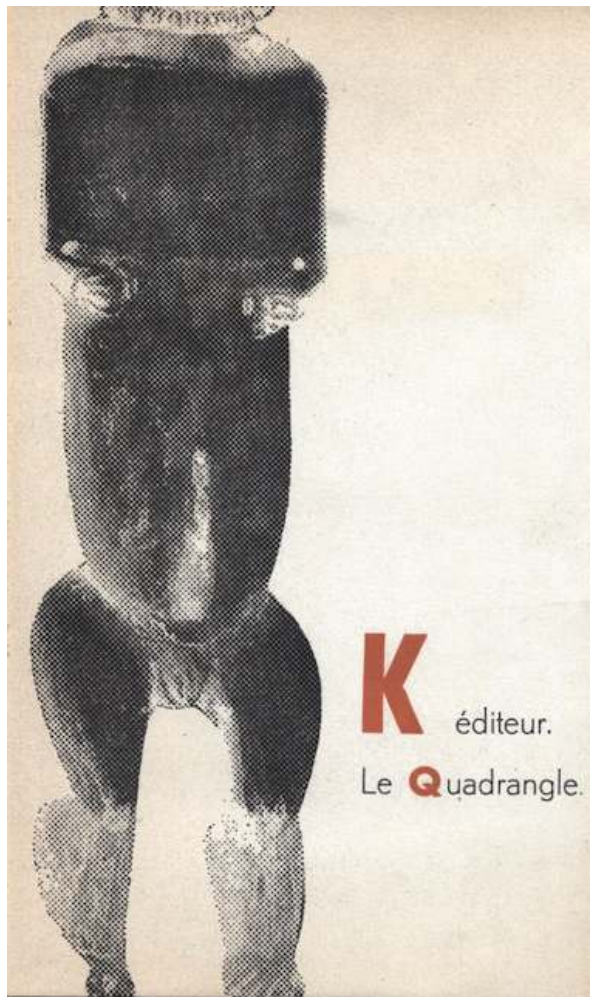

Quatrième de couverture Soleil cou coupé, 1948

Parmi les nombreux poèmes amputés de la réédition de Cadastre on trouve «Le Coup de couteau du soleil dans le dos des villes surprises». Que le titre se lise comme celui d'un tableau de Max Ernst est normal chez notre poète en 1948. Selon Pierre Laforgue « dans [« Le coup de couteau... »], la référence révolutionnaire est de nature apocalyptique, ce qui n'empêche pas la révolution de se produire une fois les sceaux brisés: "nous fondîmes sur demain avec dans nos poches le coup de couteau très violent du soleil dans le dos des villes surprises ». Ces « villes surprises » et « le coup de couteau très violent du soleil » rappellent respectivement «les villes mises à sac » et «la machete du plaisir rouge » du poème "Les Armes miraculeuses " ${ }^{8}$. Le texte se développe selon les normes de la métaphore filée décrite au début des années 1970 par Michael Riffaterre et Laurent Jenny'. Une couverture narrative empruntée au livre de l'Apocalypse qui clôt le Nouveau Testament est systématiquement interrompue par des métaphores surréalistes qui ouvrent sur l'imaginaire. Celui-ci envisage une transformation du monde par un 
«saisissement» de type spirituel qui inaugurera une nouvelle ère pour les nègres de la diaspora. Un seul poème de ce recueil mentionne «la Révolution »; c'est "CouteauxMidi » que Césaire donna à André Breton pour le catalogue de l'exposition internationale du surréalisme en $1947^{10}$. Il est manifeste que le jeune député de la Martinique tenait à maintenir simultanément, et dans un discours poétique exceptionnel, son communisme et son surréalisme. Si les intellectuels du PCF. - Aragon en tête - se sont montrés soupçonneux, André Breton, lui, n'a jamais répudié son ami martiniquais. Ils ont entretenu des relations amicales jusqu'à la mort de Breton en 1966.

Après la collaboration avec le peintre surréaliste Hans Hartung, dont une gravure originale a orné la première et quatrième de couverture de Soleil cou coupé, c'est Pablo Picasso qui a collaboré avec Césaire pour illustrer Corps perdu en 1950. C'est en vérité un drôle de phénomène bibliophilique que l'édition princeps, tirée à 207 exemplaires numérotés, plus douze hors commerce. Nos deux artistes communistes n'étaient lus que d'une poignée de riches collectionneurs.

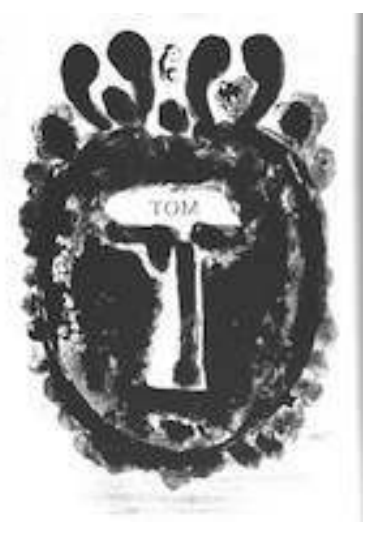

Illustration de Picasso

Les gens cultivés qui partageaient les idées de Césaire devaient attendre encore une dizaine d'années avant de faire connaissance avec le cinglant «Mot»: «le mot nègre / tout pouacre de parasites / le mot nègre / tout plein de brigands qui rôdent... »" Le dernier des dix poèmes de ce recueil, "Dit d'errance " s'ouvre sur une plainte que partagera légèrement plus tard Derek Walcott, poète de Sainte-Lucie et futur lauréat Nobel: «Tout ce qui jamais fut déchiré / en moi s'est déchiré... »" Entre-temps des interventions régies par une motivation politique auront modifié corps perdu et dévasté Soleil cou coupé. La suite de cet article expliquera pourquoi on méconnaît aujourd'hui l'originalité du poète ainsi que ses liens avec d'autres grands de la poésie du demi-siècle. Dans l'entretien avec le magazine Lire cité en exergue Césaire a avoué « je me suis parfois servi de la politique pour alimenter ma poésie. » Il ne disait que la stricte vérité... De 1939 à 1950 Aimé Césaire a créé une persona prophétique, voire messianique qu'il a bouleversée au milieu des années 1950. (à suivre) 


\section{NOTES}

1. Aimé Césaire, «Où que j'aille je reste un nègre déraciné des Antilles ", Lire $\mathrm{n}^{\circ} 87$ (novembre 1982).

2. Aimé Césaire, Poésie, Théâtre, Essais et Discours, sous la direction d'Albert James Arnold, collection Planète Libre, CNRS-Éditions, Paris, 2014, 1804 p.

3. On peut prendre connaissance de cette nouvelle lecture du poème dans The Original 1939 Notebook of a Return to my Native Land, édité en bilingue chez Wesleyan University Press en 2013 dans la traduction de Clayton Eshleman et de l'auteur de ces lignes.

4. PTED, p. 87.

5. Aimé Césaire, entretien réalisé par J.W. Garrett et J. Sorel pour les Archives Sonores de la littérature noire de Radio France Internationale en 1981, extraits reproduits par K. Véron et T. A. Hale dans Les Écrits d'Aimé Césaire, t. 2, H. Champion, Paris, 2013, p. 535.

6. P. LAFORGUE, Les Armes miraculeuses d'Aimé Césaire, Foliothèque Gallimard, 2009 ; P. VILAR, Les Armes miraculeuses d'Aimé Césaire, Le Cippe/Zoé, Bienne/Genève, 2008.

7. Dans le lexique de Césaire, le mot « apocalypse » est toujours positif, une « révélation » de type biblique. Le volcanisme de notre poète, qui se disait volontiers "péléen », est du même ordre. Loin d'être la catastrophe des gros titres du journal, l'apocalypse figurait le saisissement envisagé par Frobenius et qui tenait lieu de révolution dans la pensée de Césaire tout au long des années 1940.

8. P. Laforgue, PTED, pp. 370-371.

9. Michael Riffaterre, «Semantic Incompatibilities in Automatic Writing », About French Poetry from Dada to "Tel Quel ", Wayne State University Press, Detroit, 1974 ; Laurent Jenny, «La Surréalité et ses signes narratifs », Poétique, $n^{\circ} 16,1973$, pp. 499-520.

10. P. Laforgue, PTED, p. 376.

11. Césaire, « Mot », PTED, p. 491.

12. Césaire, «Dit d'errance », PTED, p. 508. Voir, de Derek Walcott, «A Far Cry from Africa »: "Where shall I turn / divided to the vein?»

\section{AUTEUR}

\section{ALBERT JAMES ARNOLD}

Professeur émérite, université de Virginie (USA) 